\title{
High-resolution endomicroscopy with a spectrally encoded miniature objective
}

\author{
Hamin Jeon, ${ }^{1}$ Michal E. PaWlowski, ${ }^{1}$ And Tomasz S. TKaczyK ${ }^{1,2,{ }^{*}}$ \\ ${ }^{1}$ Department of Bioengineering, Rice University, 6100 Main Street, Houston, TX 77005, USA \\ ${ }^{2}$ Department of Electrical and Computer Engineering, Rice University, 6100 Main Street, Houston, TX \\ 77005, USA \\ *ttkaczyk@rice.edu
}

\begin{abstract}
Fiber bundle endomicroscopy techniques have been used for numerous minimally invasive imaging applications. However, these techniques may provide limited spatial sampling due to the limited number of imaging cores inside the fiber bundle. Here, we present a custom-fabricated miniature objective that can be coupled to a fiber bundle and can overcome the fiber bundle's sampling threshold by utilizing the spectral encoding concept. The objective has an NA of 0.3 and an outer diameter of $2.4 \mathrm{~mm}$, and can yield a maximum spatial resolution of $2 \mu \mathrm{m}$. The objective has been validated against a USAF resolution target and ex vivo tissue samples, and as a result yielded images with higher resolution and more details after the spectral encoding concept was employed.
\end{abstract}

(C) 2019 Optical Society of America under the terms of the OSA Open Access Publishing Agreement

\section{Introduction}

Optical fiber bundles have been used to relay images onto a variety of diagnostic devices due to their unique characteristics. They are compact in size, ranging from hundreds of $\mu \mathrm{m}$ to a few $\mathrm{mm}$ in diameter [1], mechanically flexible [2], and readily available. These features have made the optical fiber a key component in many minimally invasive endoscopic systems, as it can provide a means to access various cavities in the body without causing a significant amount of tissue damage. One particular area of application includes cancer diagnostics. Cancerous lesions are commonly detected by methods such as needle biopsy and excisional biopsy [3-6]. However, these methods involve the removal of tissue and can be risky, timeconsuming, and expensive [6-8]. Thus, as an alternate method of cancer diagnostics, optical biopsy approaches that use fiber optics have been developed. Specifically, such fiber based approaches have been tested for the detection of ovarian cancer tumor nodules, Barrett's esophagus, cervical neoplasia, and oral neoplasia [9-12]. Due to its aforementioned characteristics, the optical fiber bundle has also been used in numerous confocal endoscopic systems for in vivo tissue imaging applications [13-16]. Fiber bundle based imaging systems, however, suffer from limited sampling. This is due to the fact that, for the fiber bundle to be flexible enough for in vivo imaging applications, there is an inherent compromise to the number of fiber cores and, thus, there is a trade-off between flexibility, sufficient sampling, and field of view (FOV). Commonly used fiber bundles (containing 10,000-60,000 cores), although they may be flexible, often under-sample morphological features of cells or subcellular architectures, making them difficult to have clinically practical FOV and resolution.

Improving sampling is important to accurately assess morphological features in applications such as cancer diagnostics, monitoring of brain activity, and others. For this reason, numerous attempts have been made in the past to overcome the sampling problems posed by the fiber bundle. Kyrish et al. shifted the sample and the fiber bundle relative to each other by a distance comparable to a sub-fiber core size at a time. This way, the sample was oversampled, which resulted in a lateral resolution improvement [17]. This method, however, requires actuators at the distal end of the fiber, which can be difficult to miniaturize 
and, thus, may prevent minimally invasive imaging. Shin et al. used a single-mode fiber instead of a fiber bundle and employed a scanning mechanism using a two-axis scanning mirror at the fiber's distal end [18]. However, using a scanning method can slow down the image acquisition process, which may not be ideal for in vivo imaging applications. Lee et al. superimposed multiple images that were obtained by laterally moving the fiber bundle in $\mathrm{x}$ and y directions [19]. The method yielded an image that was free of fiber bundle patterns, but no evidence of resolution improvement was shown. Languirand et al. rapidly dithered the fiber bundle to obtain information in the gap space between fiber cores [20]. However, this method can yield inconsistent resolution improvement results, as the motion of the fiber bundle resulting from the dithering process can be random. Chang et al. attached a two-axis piezoelectric scanner at the distal end of the fiber bundle and scanned the fiber laterally [21]. The resulting images after scanning were stitched together to yield a reconstructed image. As a result, the fiber bundle pattern artifact was reduced in the reconstructed image, but no evidence of resolution improvement was demonstrated. Vyas et al. shifted the fiber bundle in a precise manner using a miniaturized piezoelectric scanner to collect images that are spatially offset from each other [22]. The images were then combined together to yield a super resolution image. While the resolution improvement was clearly demonstrated in the paper, the size of the distal optics $(\sim 5 \mathrm{~mm})$ is more suitable for large instrument channels of endoscopic systems.

Besides the methods mentioned so far, which rely on a mechanical means to increase sampling of a fiber bundle, there have been attempts to use computational means to overcome the fiber bundle's sampling as well. For instance, Shao et al. used an inverted forward model by which they computed low resolution images using fiber core mapping calculated from the point spread function of a single fiber [23]. Then, a maximum a posteriori estimate of a high resolution image using conjugate gradient descent was calculated. Ravì et al. used a method of training deep neural networks (DNNs) [24]. Specifically, the authors created models that were trained using a pair of estimated high resolution images and synthetic low resolution images. Then, using such models, a high resolution image with improved quality was obtained. This approach may be, however, limited to the applications that can provide a database of images for the models to be trained on.

In this paper, we present a custom-designed imaging configuration that takes advantage of the aforementioned strengths of the fiber bundle, but does not suffer from under-sampling. The configuration combines a custom-fabricated miniature objective with an optical fiber (FIGH-30-650S, Fujikura Ltd.) and employs the spectral encoding method [25] to exceed the sampling potential of the fiber bundle. To the best of our knowledge, this is the first configuration that includes an integrated miniature probe utilizing dispersion to improve the lateral resolution of a fiber bundle based imaging system without requiring any moving components. The details of the spectral encoding concept are carefully described in our previous proof-of-concept publication [25]. While using this concept, in this article, we focus on integrating the method into an endoscope format, system integration, and testing. The custom-fabricated, miniature objective has a diameter of $2.4 \mathrm{~mm}$ and provides a spatial resolution of $2 \mu \mathrm{m}$ in the direction of dispersion when coupled with the optical fiber bundle. The objective contains a prism, which disperses an image of the sample onto the fiber bundle's distal face. As a result, each fiber core inside the fiber bundle captures spectrally encoded signals, each of which is from a different spatial location. The optical fiber works as an imaging conduit and relays these wavelength-coded signals onto the Image Mapping Spectrometer (IMS), which outputs data that is later reconstructed into a 3D data cube $(\mathrm{x}, \mathrm{y}, \lambda)$. The $3 \mathrm{D}$ data cube is subsequently broken down into $\lambda$ number of spectral channels, which are ultimately combined into a single high-resolution image using a custom MATLAB reconstruction algorithm.

The proposed optical system has three major advantages compared to other competing imaging modalities. First, it does not require any scanning apparatus, which simplifies its 
design and enables it to be more easily miniaturized. Secondly, the proposed system includes a custom-designed miniature objective as opposed to a commercial gradient index (GRIN) lens. While GRIN lens based systems [26] suffer from strong spherical and chromatic aberrations [27], the proposed system can be corrected against such aberration errors through customized design optimization step. Lastly, for systems that are fiber bundle limited, the sampling (and also resolution) can be improved by increasing magnification, although this comes at a price of FOV loss [28]. However, the proposed configuration can improve the system sampling by a factor of two without the need to change magnification and, therefore, can maintain a relatively large FOV.

\section{Development of the spectrally encoded miniature objective}

\subsection{Optical design of the objective}

The miniature objective was custom designed with the optical design software, ZEMAX (Radiant Zemax, LLC., Redmont, WA, USA), following the specifications listed in Table 1 below.

Table 1. Summary of optical parameters for the objective

\begin{tabular}{|c|c|}
\hline Object Space NA & 0.3 \\
\hline Magnification & -2.5 \\
\hline Image Space NA & 0.12 \\
\hline Working Distance & $150 \mu \mathrm{m}$ \\
\hline Wavelength Range & $515-570 \mathrm{~nm}$ \\
\hline Field of View (FOV) & $290 \mu \mathrm{m}$ \\
\hline Clear Aperture & $1.69 \mathrm{~mm}$ \\
\hline Telecentricity & Object space \\
\hline
\end{tabular}

An object space NA of 0.3 yields a diffraction limited resolution of around $1.1 \mu \mathrm{m}$ at the central wavelength of $543 \mathrm{~nm}$. The objective is designed to work with the fluorescent dye proflavine, which stains cell nuclei and whose emission full width half maximum spectrum covers the range of 515- $570 \mathrm{~nm}$ [25]. The objective's working distance of $150 \mu \mathrm{m}$ allows it to be used in close proximity to tissue and to work through 0 thickness coverslips. The optical elements are designed to be fitted inside a hypodermic tube with an outer diameter of 2.41 $\mathrm{mm}$ and an inner diameter of $2.16 \mathrm{~mm}$. The objective's clear aperture of $1.69 \mathrm{~mm}$ is smaller than the inner diameter of the tube.

The objective's lens prescription data is listed in Table 2 and its optical layout is shown in Fig. 1. The objective consists of two Poly(methyl methacrylate) (PMMA) singlet lenses, one doublet lens consisting of polystyrene and PMMA, and one zinc sulfide $(\mathrm{ZnS})$ prism. PMMA and polystyrene were chosen as lens materials since they provide high transmission and low autofluorescence in the proposed wavelength range [27]. They are also easily machinable, especially for fabricating aspheric surfaces, which effectively correct for spherical aberrations. ZnS, a polycrystalline material, was chosen as the prism material due to its high refractive index $(\mathrm{n}=2.39$ at $543 \mathrm{~nm})$ and high dispersion. The lenses making up the doublet lens were fixed together with NOA 61 optical glue (Norland, New Jersey, USA). The front surface of the prism has 4-degree angle, allowing it to disperse light over a distance of approximately $8 \mu \mathrm{m}$. The dispersion distance is long enough to cover at least the core-to-core distance of the fiber bundle. The miniature objective is designed to be immersed in saline solution. Note that the objective design was performed with seawater (see Table 2), a standard 
optical material of ZEMAX library. The optical properties of this material correspond well with those of saline solution.

Table 2. Lens prescription for the miniature objective

\begin{tabular}{|l|c|c|c|c|c|c|c|}
\hline Surface & Comment & $\begin{array}{c}\text { Radius } \\
(\mathrm{mm})\end{array}$ & $\begin{array}{c}\text { Thickness } \\
(\mathrm{mm})\end{array}$ & Material & $\begin{array}{c}\text { Semi- } \\
\text { diameter } \\
(\mathrm{mm})\end{array}$ & $\begin{array}{c}\text { Conic } \\
6^{\text {th }} \text { Order } \\
\text { Term }\end{array}$ \\
\hline OBJ & Tissue & Infinity & 0.150 & Seawater & 0.145 & 0.000 & - \\
1 & Coverslip & Infinity & 0.100 & D263M & 0.179 & 0.000 & - \\
2 & Lens 1 & Infinity & 1.750 & PMMA & 0.214 & 0.000 & - \\
3 & - & -1.096 & 0.200 & - & 0.543 & -0.297 & - \\
4 & Lens 2 & 2.031 & 1.450 & Polystyrene & 0.558 & -1.389 & $4.028 \mathrm{E}-3$ \\
5 & Glue & 0.480 & 0.100 & NOA61 & 0.445 & -1.076 & - \\
6 & Lens 3 & 0.833 & 1.500 & PMMA & 0.445 & -1.633 & - \\
7 & - & -4.753 & 0.530 & - & 0.526 & -12.264 & - \\
8 & Prism & 200 & 1.700 & ZnS & 0.584 & 0.000 & - \\
9 & - & 120 & 0.300 & - & 0.698 & 0.000 & - \\
10 & Lens 4 & 2.666 & 3.550 & PMMA & 0.738 & -0.595 & - \\
11 & - & -3.812 & 2.429 & - & 0.846 & -9.636 & - \\
IMA & Fiber bundle & Infinity & - & - & 0.759 & 0.000 & - \\
\hline
\end{tabular}

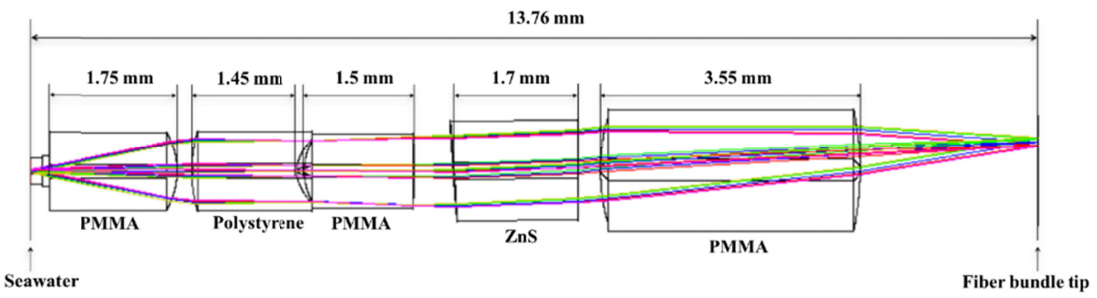

Fig. 1. Optical layout of the objective with rays from the $\mathrm{x}$-axis fields.

Nominal performance of the objective was evaluated with spot diagrams and Modulation Transfer Function (MTF) plots for the central wavelength of $543 \mathrm{~nm}$. Overall resolution of the presented system is limited by the fiber bundle and is driven by its core-to-core distance (Nyquist sampling criteria). Thus, the overall resolution in the objective's imaging space is two times the core-to-core distance, or $2 \times 3.2$ microns $=6.4$ microns. In the case where the fill factor is different than $50 \%$ (diameter of the core / diameter of the fiber), the contrast of detected features will change. In our case the core is 2.0 microns $(62.5 \%$ of the fiber diameter) which would lower the contrast and features of 6.4 microns are more difficult to visualize. The spectral encoding method can improve the sampling of the system twice and in detector limited imaging this theoretically can be improved to 3.2 microns (image space), while most likely resolvable features will be at 4 microns $(62.5 \%$ fill factor). Thus, if the nominal performance of the objective design met this limit, we considered it to be satisfactory. Also, due to the asymmetry caused by the prism in the system, fields on both sides of $\mathrm{x}$-axis and $\mathrm{y}$-axis were analyzed. The spot diagrams and MTF plots are shown below. 
a)

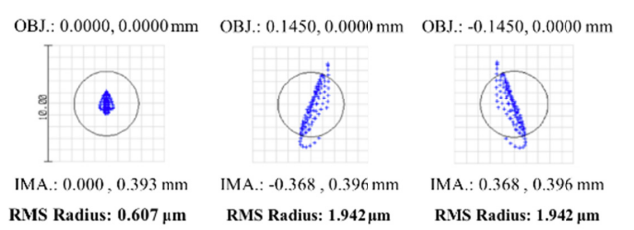

b)

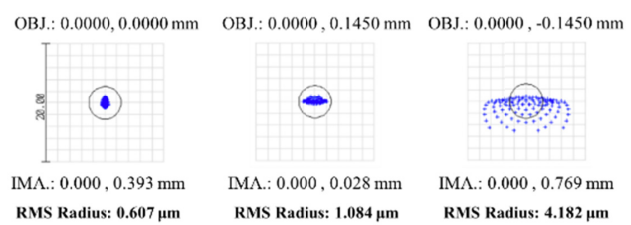

Fig. 2. (a) Spot diagrams for the fields on the $x$-axis at $543 \mathrm{~nm}$ wavelength. (b) Spot diagrams for the fields on the $y$-axis at $543 \mathrm{~nm}$ wavelength.

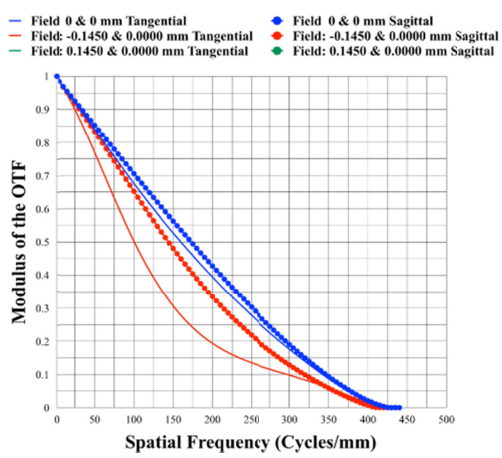

b)

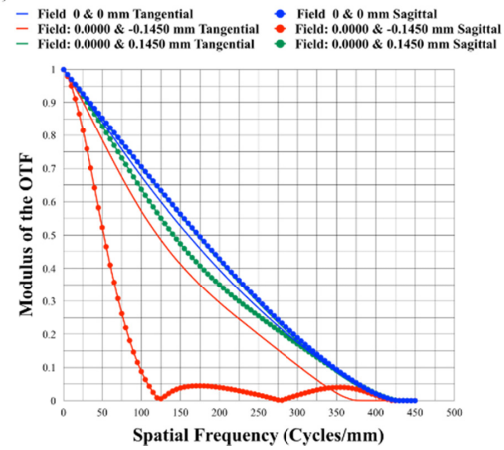

Fig. 3. (a) MTF plots for the fields on the $x$-axis at $543 \mathrm{~nm}$ wavelength. (b) MTF plots for the fields on the y-axis at $543 \mathrm{~nm}$ wavelength.

According to the spot diagrams shown in Fig. 2, the expected spatial resolution of the objective at all fields except for the negative edge of the y-axis field is higher than the expected system resolution of $3.2 \mu \mathrm{m}$. A decrease in performance towards the edge of the field is expected and the overall performance depicted by the spot diagrams was considered sufficient. Based on the MTF plots shown in Fig. 3, the objective exhibited near diffractionlimited performance for all rays except for the sagittal ray at the negative edge of the y field. Again, lower performance toward the edge of the field was expected, further due to the presence of the prism. The prism's vertically dispersive characteristic leads to the objective's asymmetric performance across fields on the y-axis. This causes a significant drop in performance at the negative edge of the y-axis field, while the objective's performance at the positive edge of the $y$-axis field is fair.

\subsection{Tolerance optimization}

Manufacturability of the objective design was evaluated in ZEMAX. The complete process of lens development has been characterized prior to the design step (see Table 3) for both the fabrication and assembly of the objective. Thus, the objective design process was performed using these input tolerance values. Specifically, the miniature objective's performance was evaluated using root sum square (RSS) and Monte Carlo analysis methods based on its root mean square (RMS) spot radius.

Table 3. Expected tolerances for the miniature objective

\begin{tabular}{|c|c|c|c|c|c|c|c|c|c|}
\hline Parameter & $\begin{array}{c}\text { Radii } \\
(\mathrm{mm})\end{array}$ & $\begin{array}{c}\text { Thickness } \\
(\mathrm{mm})\end{array}$ & $\begin{array}{c}\text { Element } \\
\text { Decenter } \\
(\mathrm{mm})\end{array}$ & $\begin{array}{c}\text { Element } \\
\text { Tilt } \\
\left({ }^{\circ}\right)\end{array}$ & $\begin{array}{c}\text { Surface } \\
\text { Decenter } \\
(\mathrm{mm})\end{array}$ & $\begin{array}{c}\text { Surface } \\
\text { Tilt } \\
(\mathrm{mm})\end{array}$ & $\begin{array}{c}\text { Irregularity } \\
\text { (Fringes) }\end{array}$ & $\begin{array}{c}\text { Abbe } \\
(\%)\end{array}$ & $\begin{array}{c}\mathrm{N} \\
(\text { a.u. })\end{array}$ \\
\hline Value & \pm 0.01 & \pm 0.04 & \pm 0.025 & \pm 0.2 & \pm 0.025 & \pm 0.02 & \pm 0.2 & \pm 2 & \pm 0.004 \\
\hline
\end{tabular}


When the objective design was assessed based on the RMS spot radius criterion using the RSS algorithm, the design yielded a nominal performance of $1.44 \mu \mathrm{m}$, a change in performance of $1.58 \mu \mathrm{m}$, and a total performance of $3.02 \mu \mathrm{m}$. The estimated total performance of $3.02 \mu \mathrm{m}$ is higher than the required performance of $3.2 \mu \mathrm{m}$ and, thus, was considered satisfactory. Based on 10,000 Monte Carlo simulations, there was a $90 \%$ chance of manufacturing an objective with an RMS spot radius below $3.2 \mu \mathrm{m}$. Based on the satisfactory tolerance optimization results from both the RSS algorithm and Monte Carlo simulations, the objective design was considered sufficient even when manufacturing and assembly processes were taken into account.

\subsection{Fabrication}

As a first step, the objective was modeled using SolidWorks (Dassault Systèmes). The modeled design included detailed features such as precision spacer features, which were added to provide accurate spacing between lens surfaces. An example of these features is shown in Fig. 4(a). After modeling the objective, precursors for the lenses were cut from rods of raw material. Spatial coordinates needed to cut the lenses were set using the software, NanoCAM 2D (Moore Nanotechnology Systems, LLC, Swanzey, New Hampshire). After the cutting coordinates were determined, the surfaces were cut using the in-house single point diamond turning (SPDT) machine as illustrated in Fig. 6(a). To make the doublet, a drop of NOA 61 glue was applied onto a concave surface of the polystyrene lens. After using a vacuum chamber to remove any bubbles inside the glue droplet, the convex surface of the PMMA lens was placed onto the concave surface of the polystyrene lens. The surfaces came in contact concentrically based on a feature already cut into the surfaces as shown in Fig. 4(b). The glue was then cured using ultraviolet light. To fabricate the prism, an in-house polishing machine was used, depicted in Fig. 6(b). The machine has an angle control knob, which was used to polish one surface of the prism at 4-degree angle. After both surfaces of the prism were polished, an in-house white light interferometer was used to verify that the prism surfaces were of optical quality. To minimize the amount of stray light that can enter the lens surfaces, optical stops were also incorporated in the final objective assembly, which are shown as the black surfaces in Fig. 4(c).

a)

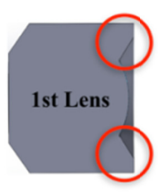

b)

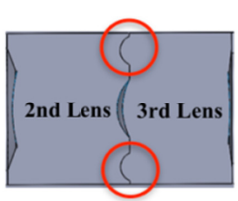

c)

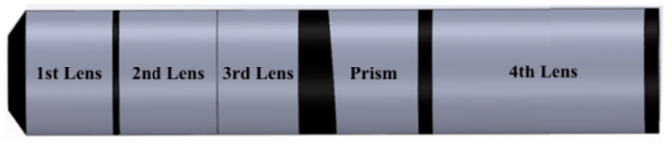

Fig. 4. (a) Features that are circled red are spacing components that are embedded into a lens to ensure the proper distance between surfaces. (b) Features circled red ensure that two surfaces come in contact concentrically. (c) Assembly of all optical elements.

The fabricated optical elements were assembled inside a stainless steel hypodermic tube with an outer diameter of $2.41 \mathrm{~mm}$ and an inner diameter of $2.16 \mathrm{~mm}$. A cross-sectional view of the assembled objective is shown in Fig. 5. Also, the fabricated optical elements were placed on a U.S. penny for size comparison, as depicted in Fig. 6(c). After assembling the elements inside the hypodermic tube, a cover slip was attached onto the distal side of the objective to protect the objective's front surface from damage. 


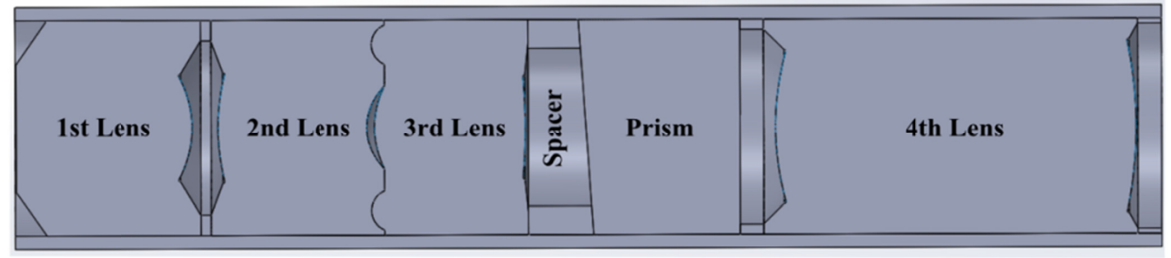

Fig. 5. SolidWorks cutaway of the assembled miniature objective.

a)

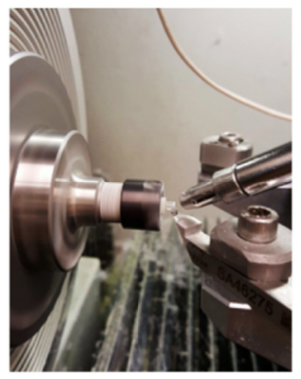

b)

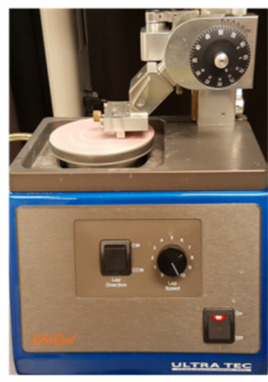

c)

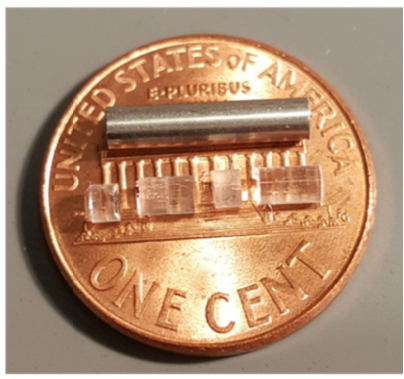

Fig. 6. (a) Processing an optical element by using the SPDT machine. (b) Fabrication of the prism using the in-house polishing machine. (c) Components of the miniature objective placed on a U.S. penny for size comparison.

\subsection{Objective performance}

Lateral resolution of the objective was evaluated using a standard 1951 USAF (United States Air Force) high resolution target. The optical setup used to assess the objective's performance is shown in Fig. 7. For the light source, a white-light LED (MCWHL2, Thorlabs, USA) was used and a 10X, 0.25 NA commercial objective (Zeiss, Germany) and Zeiss tube lens were used to relay the image of the resolution target onto a CCD camera (Lumenera Corporation, Canada). Chromatic aberrations caused by the prism's dispersion was reduced with $10 \mathrm{~nm}$ bandpass filters (Edmund Optics, USA) centered at different wavelengths. Results of the assessment are depicted in Fig. 8. Based on Fig. 8, the objective was able to resolve the smallest features on the target, which are bars from group 9, element $3(645 \mathrm{lp} / \mathrm{mm})$, in the proposed wavelength range.

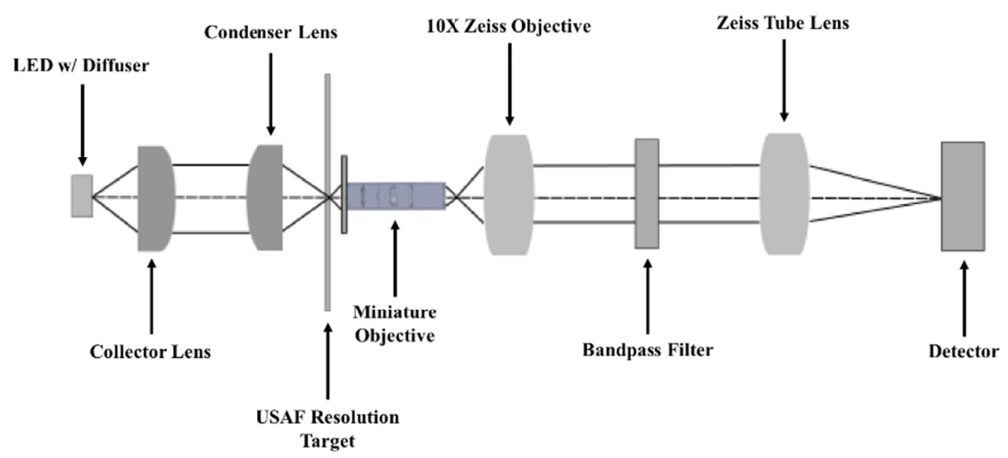

Fig. 7. Optical setup used to evaluate the lateral resolution of the objective. 


\section{Biomedical Optics EXPRESS}
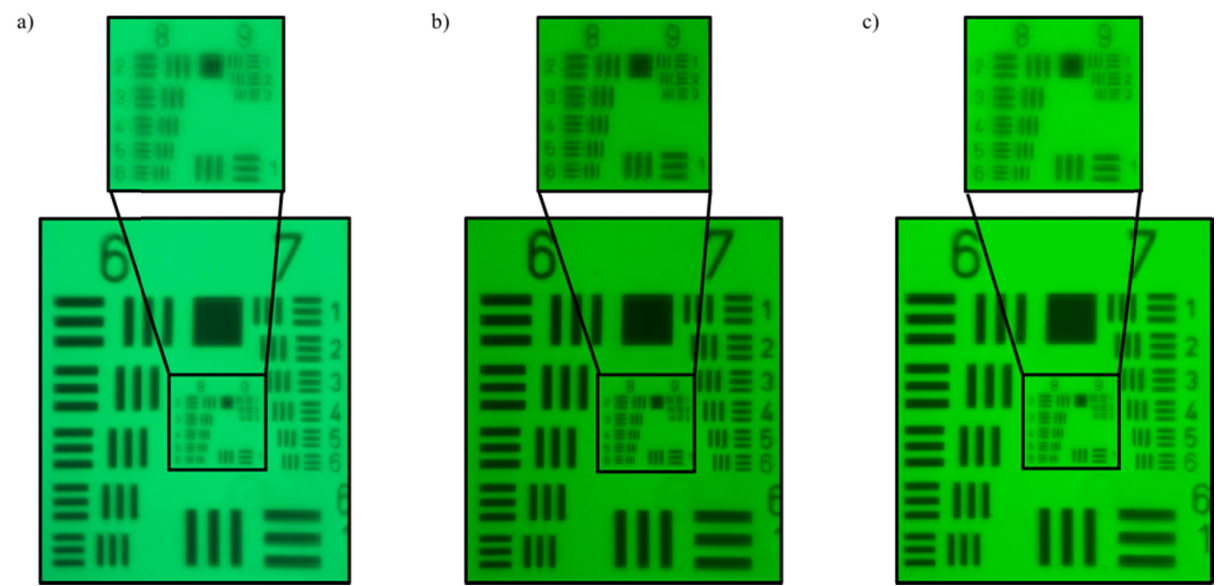

Fig. 8. (a) USAF target image taken with a $10 \mathrm{~nm}$ bandpass filter centered at $515 \mathrm{~nm}$, (b) 540 $\mathrm{nm}$, and (c) $568 \mathrm{~nm}$. All images have been contrast enhanced for visualization purposes.

\section{Enhancing image quality through the spectral encoding concept}

\subsection{Imaging results with an USAF resolution target}

The optical setup shown in Fig. 9 was used to verify the resolution improvement through the spectral encoding method [25]. The miniature objective captures signal from the resolution target and the prism inside the objective disperses the signal onto the distal face of the fiber bundle.

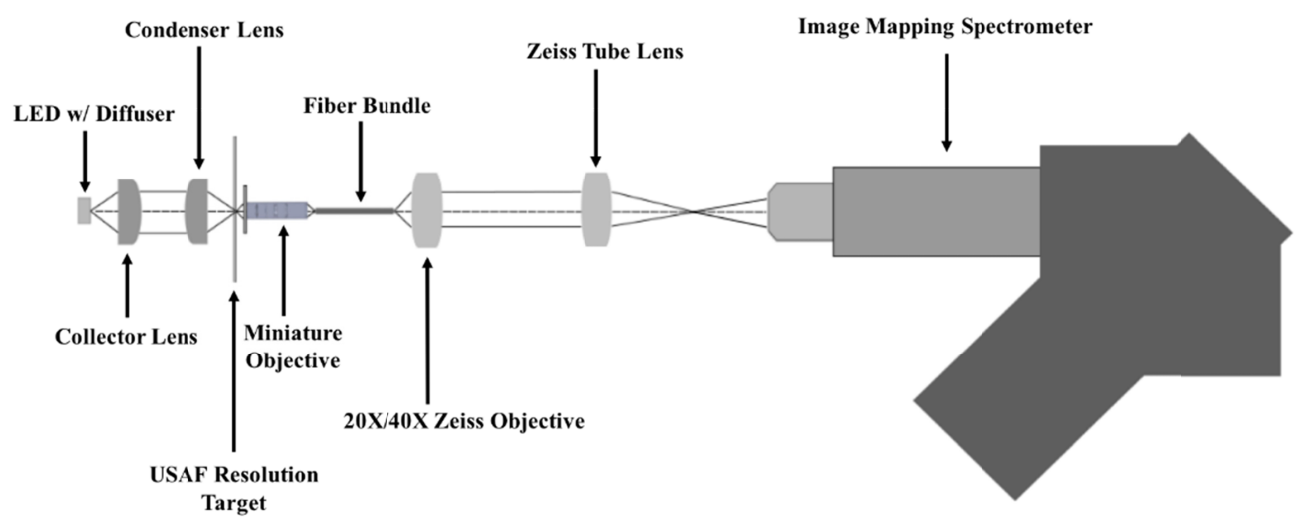

Fig. 9. Optical setup used to verify the spectral encoding concept with USAF resolution target.

The prism was oriented in a way to provide dispersion in the horizontal direction. As a result of the dispersion, each core of the fiber bundle receives spatially offset signals that are spectrally encoded with different wavelengths. These signals are then captured by the Image Mapping Spectrometer (IMS), which was custom-built in our lab [29]. The IMS is a snapshot spectrometer that has high light efficiency and, thus, is ideal for imaging fluorescent samples that yield low light signal [25]. It acquires $356 \times 336$ spatial samples and 21 spectral samples in the 515-570 $\mathrm{nm}$ wavelength range. Therefore, if coupled with a fiber bundle with 30,000 cores, $30,000 \times 21$, or 630,000 sampling points can be acquired.

The IMS returns data that can be processed by a custom calibration algorithm [29] to yield a 3D data cube $(x, y, \lambda)$. The $3 \mathrm{D}$ data cube and a custom MATLAB reconstruction algorithm are used offline after the imaging experiment to obtain a high resolution image that demonstrates resolution improvement. The reconstruction algorithm consists of three major 
steps: 1) extracting information from fiber cores, 2) calculating the spatial offset between channels, and 3) combining spectral channel images. A detailed explanation of each step is included in the following paragraphs.

In order to extract information from fiber cores, each fiber core location is first determined by computing local maxima points, each of which corresponds to an individual fiber core. The local maxima points are calculated using MATLAB's imregionalmax.m function, which is described in Ref. 30. For the setup shown in Fig. 9 with a 20X objective, each fiber core is sampled by approximately a single detector pixel and the local maximum point is used to represent each fiber core. We also used a setup with a $40 \mathrm{X}$ objective, which provides a magnification twice that of the 20X objective setup. For such setup, each fiber core is oversampled by detector pixels and neighboring pixels of the local maximum pixel are also used to make up each core. Each fiber core's central pixel location is calculated by taking the average value of the row and column coordinates of the pixels in a fiber core, and the central pixel's intensity is calculated by taking the average intensity of all pixels in each fiber core. This central pixel, thus, represents an individual fiber core.

As a next step, the offset between spectral channels is computed using the registration algorithm described in Ref. 31. The registration algorithm computes the cross correlation of two images using discrete Fourier transforms (DFTs). The cross correlation of two images $f(x, y)$ and $g(x, y)$ is calculated by the equation:

$$
r_{f g}\left(x_{0}, y_{0}\right)=\sum_{u, v} F(u, v) G^{*}(u, v) \exp \left[i 2 \pi\left(\frac{u x_{0}}{M}+\frac{v y_{0}}{N}\right)\right]
$$

where $\mathrm{N}$ and $\mathrm{M}$ represent the image dimensions, uppercase letters $\mathrm{F}$ and $\mathrm{G}$ represent the discrete Fourier transform of their lowercase counterparts, $\left(\mathrm{x}_{0}, \mathrm{y}_{0}\right)$ represents the global coordinate translation, and $*$ denotes complex conjugation. The offset between two images can be calculated by (1) computing the product, $\mathrm{F}(\mathrm{u}, \mathrm{v}) \mathrm{G}^{*}(\mathrm{u}, \mathrm{v})$, (2) taking the inverse fast Fourier transform to calculate the cross correlation, and (3) locating the peak of the cross correlation [31]. Based on this spatial offset, information from the fiber cores of all spectral channels within the range of $515 \mathrm{~nm}$ to $570 \mathrm{~nm}$ is combined into one image. In the IMS's spectral range of 515 to $570 \mathrm{~nm}$, which spans the emission spectrum of proflavine, the dispersion distance of at least the core-to-core distance of a fiber bundle is provided, which is required to obtain a reconstructed image that exhibits resolution improvement. In this spectral range, the IMS calibration matrix provides 21 spectral images. If there are still any void spaces left after combining all the spectral images, the empty spaces are assigned with values that are calculated based on a linear interpolation method [32]. The final result using the setup from Fig. 9 and a 20X objective is shown in Fig. 10 and the final result obtained using the setup that utilizes a 40X Zeiss objective is shown in Fig. 11.
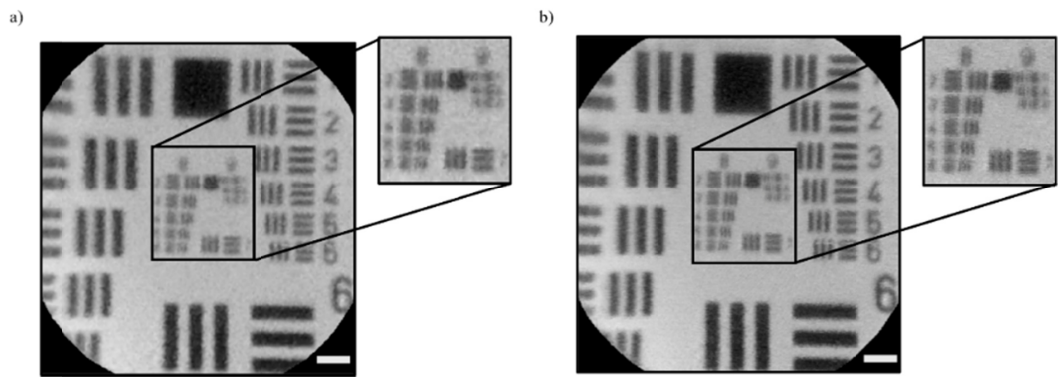

Fig. 10. Spectral encoding method results obtained with the setup shown in Fig. 9. (a) Reconstructed image using only the $543 \mathrm{~nm}$ spectral channel. (b) Reconstructed image using 21 spectral images. All images have been contrast enhanced for visualization purposes. The scale bars shown represent 20 microns. 
a)

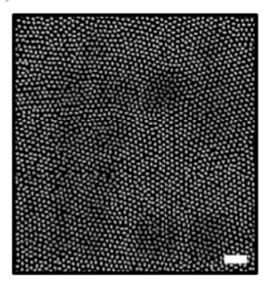

b)

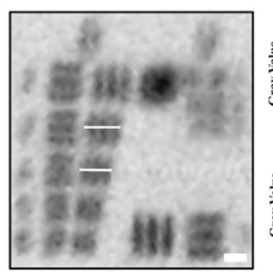

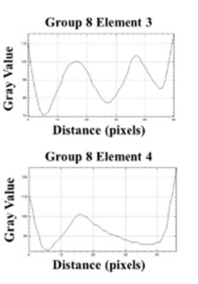

c)

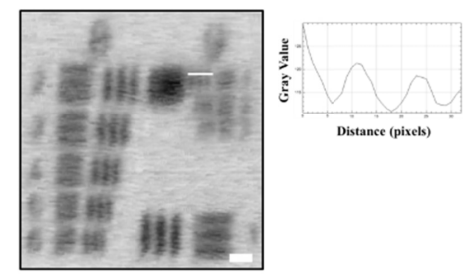

Fig. 11. Image of an Air Force Resolution target with a 40x objective in the IMS detection system. (a) Raw $543 \mathrm{~nm}$ channel image. (b) Reconstructed image using only the $543 \mathrm{~nm}$ spectral channel and line profile analysis results for the vertical features in group 8, elements 3 and 4. (c) Reconstructed image using 21 spectral images and line profile analysis result for the vertical features in group 9, element 1. All images have been contrast enhanced for visualization purposes. The scale bars in all figures represent 5 microns.

Based on Fig. 11, up to group 8, element $2(287.4 \mathrm{lp} / \mathrm{mm})$ features are seen in the raw single channel image. The resolvable features are a product of the $2.5 \mathrm{x}$ magnification and the sampling limit of the fiber bundle (note that our system is fiber bundle limited). After the reconstruction step using a single spectral channel, we are able to observe up to group 8 , element $3(322.5 \mathrm{lp} / \mathrm{mm})$ vertical features. Cross sections through the group 8, element 4 target features in Fig. 11(b) demonstrate that they do not show the expected sinusoidal pattern. The cross section in Fig. 11(c) shows that group 9, element 1 can be resolved after using the spectral encoding concept. The resolution improvement in vertical features is seen due to the fact that the dispersion was in the horizontal direction. The degree of resolution improvement shown between Fig. 11(b) and Fig. 11(c) was 1.6 times rather than the theoretically expected 2 times. We hypothesize that the difference is due to the alignment of the optical setup shown in Fig. 9 and irregular distribution of imaging cores inside the fiber bundle. In general, the sampling improvement is expected to be in only one direction. Some bi-directional improvement can be seen due to the irregular pattern of the coherent fiber bundle as seen in our prior work [25]. In this study, the 20X objective results shown in Fig. 10 , which were based on sampling that was at the edge of the Nyquist frequency, show predominantly one directional improvement. When using the $40 \mathrm{X}$ objective, during which the cores were easily visualized, some bi-directional improvement was also observed.

\subsection{Imaging results with ex vivo tissue samples}

Porcine tissue samples ( $\mathrm{J}$ and $\mathrm{J}$ Packing Company Inc., Brookshire, TX and Fisher Ham \& Meat Co, Spring, TX) were used to further validate the objective's performance. Particularly, oral cheek tissues were imaged, as their epithelial layer contained a plethora of nuclei to which proflavine readily bound to. Before imaging, the samples were stained with $0.01 \% \mathrm{w} / \mathrm{v}$ proflavine dye and any excess dye was washed afterward with $1 \mathrm{X}$ phosphate buffered saline (PBS) solution. The optical setup shown in Fig. 12 was used to image the ex vivo tissue samples. 


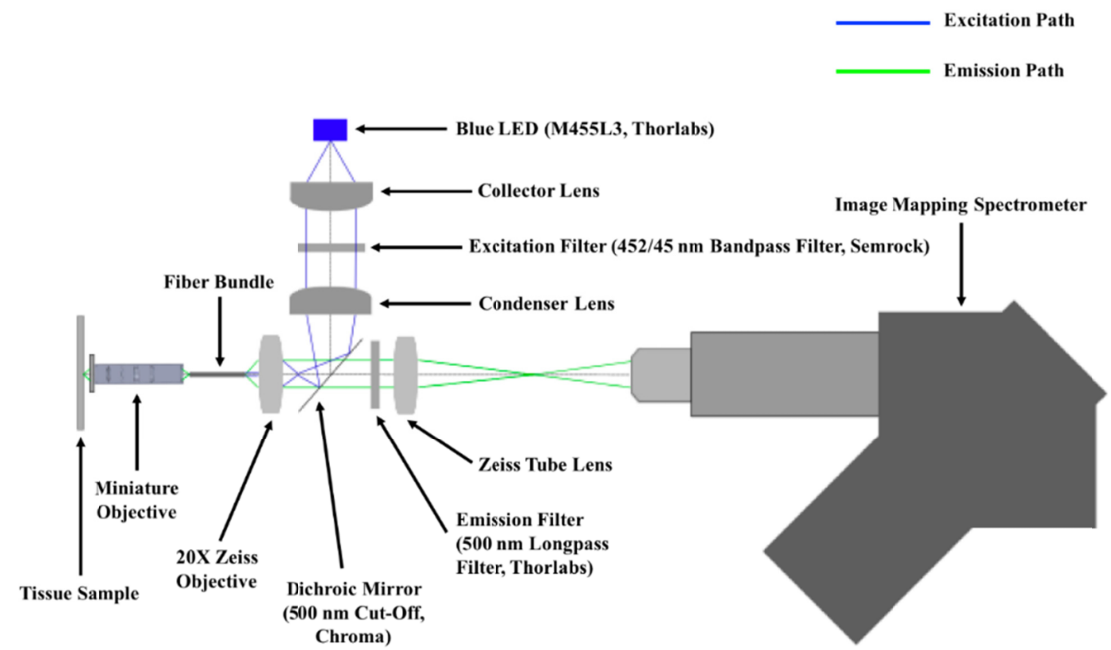

Fig. 12. Optical setup used to verify the spectral encoding concept with oral cheek samples from a pig.

The result obtained after employing the spectral encoding method is shown in Fig. 13. We also used a commercial microscope (Zeiss Primo Star microscope, Germany) to take an image of the tissue sample and compared this result to the reconstructed image, which is depicted in Fig. 14. Different areas were captured by the setup shown in Fig. 12 and the commercial microscope, respectively, due to the difficulty of imaging the same areas in two different optical setups.
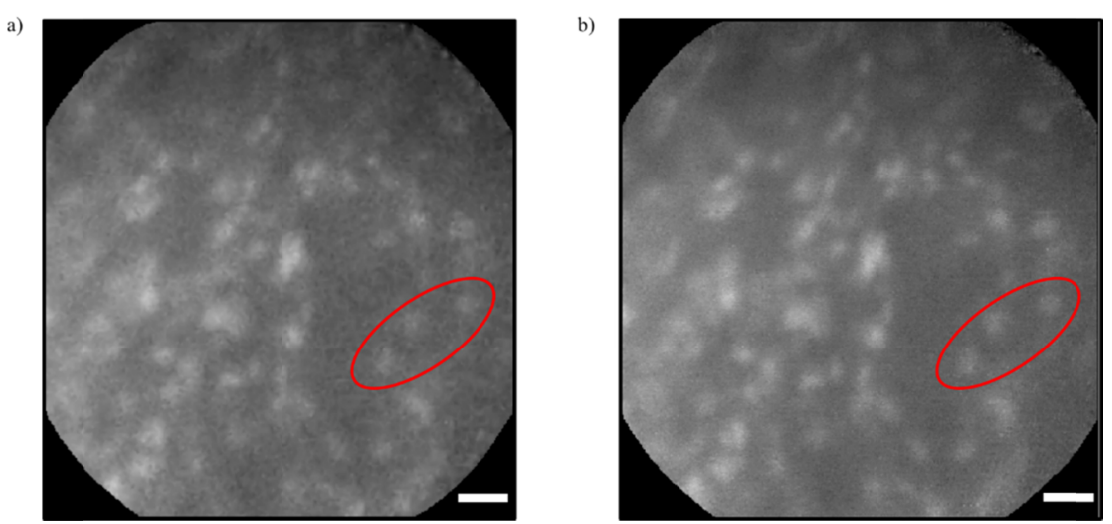

Fig. 13. Spectral encoding method results obtained using porcine oral cheek samples. (a) Reconstructed image obtained using only the $543 \mathrm{~nm}$ spectral channel. (b) Reconstructed image using 21 spectral images. All images have been contrast enhanced for visualization purposes. The scale bars shown represent 20 microns. 


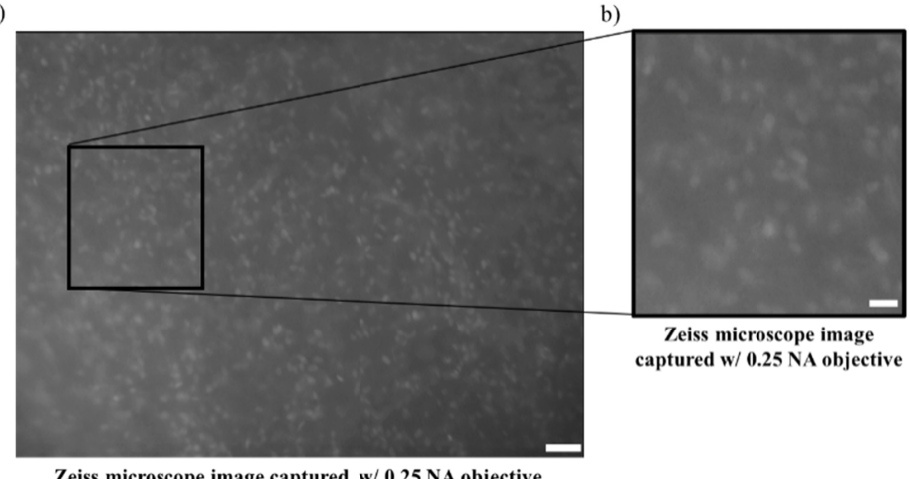

c)

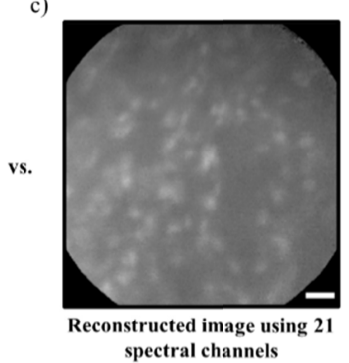

Fig. 14. Comparison between the tissue images obtained with a commercial microscope and the spectral encoding method. All images have been contrast enhanced for visualization purposes. The scale bar shown in Fig. 14(a) represents 50 microns while the scale bars shown in Fig. 14(b) and (c) represent 20 microns.

Based on Fig. 13, outlines of the cell nuclei are defined more clearly and are more visible in the reconstructed image using 21 spectral images than they are in the reconstructed image using a single spectral channel. According to Fig. 14, the overall quality of the reconstructed image using 21 spectral images is comparable to that of the image obtained with the commercial microscope.

\section{Discussion}

Based on the lateral resolution assessment of the miniature objective using the setup shown in Fig. 7, the objective could resolve the smallest features on the high resolution USAF target, which are the bars in group 9, element $3(645 \mathrm{lp} / \mathrm{mm})$. When coupled to a fiber bundle, the objective could resolve up to group 8 , element $3(322.5 \mathrm{lp} / \mathrm{mm})$ features, but, after using the spectral encoding concept, the objective could image the vertical features in group 9 , element $1(512 \mathrm{lp} / \mathrm{mm})$. Based on this result, the resolution improvement in the dispersion direction based on the spectral encoding method has been successfully demonstrated. The objective's performance has additionally been evaluated against ex vivo oral cheek tissue samples. Based on the result shown in Fig. 14, the objective yielded an image of cell nuclei whose quality was comparable to that of the image obtained with a commercial Zeiss Primo Star microscope. In the future, to better demonstrate the resolution improvement of the endoscope, we plan to perform additional imaging experiments of biological samples including colon crypts and neural cell structures, during which we will operate close to the resolution limit.

Currently, the miniature objective can provide sampling increase in a single direction, which can be disadvantageous if the biological sample of interest contains important physical features in both $\mathrm{x}$ and $\mathrm{y}$ directions. Also, the current objective's capability to operate in a single color channel limits the number of fluorescent dyes that it can work with, which can in turn limit the breadth of applications that the objective can be used for. Lastly, the reconstruction algorithm for yielding an image with higher resolution is currently used offline after collecting the raw sample images. This may prevent the objective from being used for any real-time imaging applications that might require the reconstruction process to be done online. Therefore, to address these limitations, we intend to follow up with the design and development of an objective that can provide resolution improvement in two directions and operate across a broadband wavelength range. The objective will contain custom-designed prisms that can disperse in both $\mathrm{x}$ and $\mathrm{y}$ directions, which will lead to a sampling increase in two directions. The objective will also simultaneously work with multiple dyes whose emission wavelength range can span the visible wavelength range and can be used to monitor a variety of cellular functions or image various cellular features. In addition, the 
reconstruction algorithm will be further optimized to reduce its computational cost and time to ultimately be used for in vivo real-time imaging applications.

\section{Conclusions}

In this study, a high-resolution, spectrally encoded miniature objective has been designed, fabricated, and assessed. The objective has an outer diameter of $2.4 \mathrm{~mm}$ and can easily be integrated with a system that utilizes a fiber bundle for minimally invasive imaging applications. The performance of the miniature probe was validated against a USAF resolution target and ex vivo tissue samples. As a result, the probe successfully yielded images with higher resolution and more details after the spectral encoding concept was employed without requiring any scanning components. Since the objective can obtain an image in a snapshot fashion at a video rate, optimization of the current reconstruction algorithm may allow the system to be used for real-time imaging applications in the future. As a next step, we aim to develop an in vivo minimally invasive imaging modality after further miniaturizing the distal optics and fabricating an objective with an outer diameter of approximately $1 \mathrm{~mm}$. The objective will include a disperser allowing two-dimensional encoding, which will enable us to increase sampling in both vertical and horizontal directions. The objective will also work in the broadband wavelength range, which will allow it to simultaneously work with multiple dyes.

\section{Funding}

Gulf Coast Consortia, on the IGERT: Neuroengineering from Cells to Systems (Training Fellowship); National Science Foundation (NSF) (1250104); John S. Dunn Foundation Collaboration Research Award Program; National Institutes of Health (NIH R01 CA103830).

\section{Disclosures}

Tomasz S. Tkaczyk has financial interests in Attoris LLC focusing on hyperspectral technologies.

\section{References}

1. B. A. Flusberg, E. D. Cocker, W. Piyawattanametha, J. C. Jung, E. L. M. Cheung, and M. J. Schnitzer, "FiberOptic Fluorescence Imaging," Nat. Methods 2(12), 941-950 (2005).

2. A. D. Mehta, J. C. Jung, B. A. Flusberg, and M. J. Schnitzer, "Fiber Optic in Vivo Imaging in the Mammalian Nervous System," Curr. Opin. Neurobiol. 14(5), 617-628 (2004).

3. "Types of biopsies used to look for cancer." American Cancer Society. 30 July 2015. Retrieved from https://www.cancer.org/treatment/understanding-your-diagnosis/tests/testing-biopsy-and-cytology-specimensfor-cancer/biopsy-types.html

4. Y. J. Zhang, L. Wei, J. Li, Y. Q. Zheng, and X. R. Li, "Status quo and development trend of breast biopsy technology," Gland Surg. 2(1), 15-24 (2013).

5. C. F. Loughran and C. R. Keeling, "Seeding of tumour cells following breast biopsy: a literature review," Br. J. Radiol. 84(1006), 869-874 (2011).

6. K. Shyamala, H. C. Girish, and S. Murgod, "Risk of tumor cell seeding through biopsy and aspiration cytology," J. Int. Soc. Prev. Community Dent. 4(1), 5-11 (2014).

7. C. J. Cobb, "FNA or core needle biopsy? Why not both?" CAP Today 107(November) (2007), www.captodayonline.com/Archives/pap_ngc/1107NGC_FNA.html.

8. L. Wang, "Early Diagnosis of Breast Cancer," Sensors (Basel) 17(7), 1572 (2017).

9. W. Zhong, J. P. Celli, I. Rizvi, Z. Mai, B. Q. Spring, S. H. Yun, and T. Hasan, "In Vivo High-Resolution Fluorescence Microendoscopy for Ovarian Cancer Detection and Treatment Monitoring,” Br. J. Cancer 101(12), 2015-2022 (2009).

10. T. J. Muldoon, S. Anandasabapathy, D. Maru, and R. Richards-Kortum, "High-Resolution Imaging in Barrett's Esophagus: A Novel, Low-Cost Endoscopic Microscope," Gastrointest. Endosc. 68(4), 737-744 (2008).

11. M. K. Quinn, T. C. Bubi, M. C. Pierce, M. K. Kayembe, D. Ramogola-Masire, and R. Richards-Kortum, "HighResolution Microendoscopy for the Detection of Cervical Neoplasia in Low-Resource Settings," PLoS One 7(9), e44924 (2012).

12. T. J. Muldoon, D. Roblyer, M. D. Williams, V. M. Stepanek, R. Richards-Kortum, and A. M. Gillenwater, "Noninvasive Imaging of Oral Neoplasia with a High-Resolution Fiber-Optic Microendoscope," Head Neck 9999(9999), 1-8 (2011). 
13. A. R. Rouse and A. F. Gmitro, "Multispectral imaging with a confocal microendoscope," Opt. Lett. 25(23), $1708-1710$ (2000).

14. R. T. Kester, T. S. Tkaczyk, M. R. Descour, T. Christenson, and R. Richards-Kortum, "High numerical aperture microendoscope objective for a fiber confocal reflectance microscope," Opt. Express 15(5), 2409-2420 (2007).

15. H. Makhlouf, A. F. Gmitro, A. A. Tanbakuchi, J. A. Udovich, and A. R. Rouse, "Multispectral confocal microendoscope for in vivo and in situ imaging," J. Biomed. Opt. 13(4), 044016 (2008).

16. J. Wang, H. Li, G. Tian, Y. Deng, Q. Liu, and L. Fu, "Near-infrared probe-based confocal microendoscope for deep-tissue imaging," Biomed. Opt. Express 9(10), 5011-5025 (2018).

17. M. Kyrish, R. Kester, R. Richards-Kortum, and T. Tkaczyk, "Improving Spatial Resolution of a Fiber Bundle Optical Biopsy System," Proc SPIE Int Soc Opt Eng 7558, 755807 (2010).

18. H. J. Shin, M. C. Pierce, D. Lee, H. Ra, O. Solgaard, and R. Richards-Kortum, "Fiber-Optic Confocal Microscope Using a MEMS Scanner and Miniature Objective Lens," Opt. Express 15(15), 9113-9122 (2007).

19. C. Y. Lee and J. H. Han, "Elimination of Honeycomb Patterns in Fiber Bundle Imaging by a Superimposition Method," Opt. Lett. 38(12), 2023-2025 (2013).

20. E. R. Languirand and B. M. Cullum, "Large area super-resolution chemical imaging via rapid dithering of a nanoprobe," Proc. SPIE 9487, 94870 (2015).

21. Y. Chang, W. Lin, J. Cheng, and S. C. Chen, "Compact high-resolution endomicroscopy based on fiber bundles and image stitching," Opt. Lett. 43(17), 4168-4171 (2018).

22. K. Vyas, M. Hughes, B. G. Rosa, and G. Z. Yang, "Fiber bundle shifting endomicroscopy for high-resolution imaging," Biomed. Opt. Express 9(10), 4649-4664 (2018).

23. J. Shao, W. C. Liao, R. Liang, and K. Barnard, "Resolution enhancement for fiber bundle imaging using maximum a posteriori estimation," Opt. Lett. 43(8), 1906-1909 (2018).

24. D. Ravì, A. B. Szczotka, D. I. Shakir, S. P. Pereira, and T. Vercauteren, "Effective deep learning training for single-image super-resolution in endomicroscopy exploiting video-registration-based reconstruction," Int. J. CARS 13(6), 917-924 (2018).

25. N. Bedard and T. S. Tkaczyk, "Snapshot Spectrally Encoded Fluorescence Imaging through a Fiber Bundle," J. Biomed. Opt. 17(8), 080508 (2012).

26. J. Knittel, L. Schnieder, G. Buess, B. Messerschmidt, and T. Possner, "Endoscope-Compatible Confocal Microscope Using a Gradient Index-Lens System," Opt. Commun. 188(5-6), 267-273 (2001).

27. M. Kyrish and T. S. Tkaczyk, "Achromatized Endomicroscope Objective for Optical Biopsy," Biomed. Opt. Express 4(2), 287-297 (2013).

28. L. Yang, J. Wang, G. Tian, J. Yuan, Q. Liu, and L. Fu, "Five-Lens, Easy-to-Implement Miniature Objective for a Fluorescence Confocal Microendoscope," Opt. Express 24(1), 473-484 (2016).

29. N. Bedard, N. Hagen, L. Gao, and T. S. Tkaczyk, "Image Mapping Spectrometry: Calibration and Characterization," Opt. Eng. 51(11), 111711 (2012).

30. P. Soille, Morphological Image Analysis: Principles and Applications (Springer, 1999)

31. M. Guizar-Sicairos, S. T. Thurman, and J. R. Fienup, "Efficient subpixel image registration algorithms," Opt. Lett. 33(2), 156-158 (2008).

32. S. Rupp, C. Winter, and M. Elter, "Evaluation of spatial interpolation strategies for the removal of combstructure in fiber-optic images," 2009 Annual International Conference of the IEEE Engineering in Medicine and Biology Society 3677-3680 (2009). 